\title{
Deflation-based identification of nonlinear excitations of the three-dimensional Gross-Pitaevskii equation
}

\author{
N. Boullé $\odot,{ }^{1, *}$ E. G. Charalampidis $\odot,{ }^{2, \dagger}$ P. E. Farrell $\odot,{ }^{1, *}$ and P. G. Kevrekidis ${ }^{1,3, \S}$ \\ ${ }^{1}$ Mathematical Institute, University of Oxford, Oxford OX2 6GG, United Kingdom \\ ${ }_{2}^{2}$ Mathematics Department, California Polytechnic State University, San Luis Obispo, California 93407-0403, USA \\ ${ }^{3}$ Department of Mathematics and Statistics, University of Massachusetts Amherst, Amherst, Massachusetts 01003-4515, USA
}

(Received 22 April 2020; revised 20 October 2020; accepted 22 October 2020; published 9 November 2020)

\begin{abstract}
We present a number of solutions to the three-dimensional Gross-Pitaevskii equation describing atomic BoseEinstein condensates. This model supports elaborate patterns, including excited states bearing vorticity. The coherent structures exhibit striking topological features, involving combinations of vortex rings and multiple, possibly bent vortex lines. Although unstable, many of them persist for long times in dynamical simulations. These solutions were identified by a state-of-the-art numerical technique called deflation, which is expected to be applicable to many problems from other areas of physics.
\end{abstract}

DOI: 10.1103/PhysRevA.102.053307

\section{INTRODUCTION}

The nonlinear Schrödinger (NLS) or Gross-Pitaevskii (GP) equation [1-6] is a fundamental partial differential equation that combines dispersion and nonlinearity. It has been central to a variety of areas of mathematical physics for several decades. The NLS/GP model has facilitated a universal description of a wide range of phenomena, including electric fields in optical fibers [7], Langmuir waves in plasmas [8], freak waves in the ocean [9], and Bose-Einstein condensates (BECs). In the past 25 years since the experimental realization of atomic BECs, the NLS/GP model has enabled the theoretical identification and experimental observation of a wide range of coherent structures, including (but not limited to) dark [10] and bright [11] solitary waves, two-dimensional vortical patterns and lattices [12,13] as well as vortex lines and rings [14].

The examination of three-dimensional (3D) systems has been a key frontier of recent studies in BECs. Recent theoretical advances have enabled the capturing of a number of such states [15]. Some, especially topological ones such as skyrmions, monopoles, and Alice rings $[16,17]$ have been of particular interest since the early exploration of BECs, while others such as knots [18] have been studied more recently. In this manuscript, we apply a powerful numerical technique called deflation [19-21] to identify multiple solutions of the 3D NLS/GP equation.

Many of the solutions obtained by this process are identifiable as nonlinear extensions of solutions of the linear limit of the problem, or as bifurcations therefrom. Yet other solutions are highly unexpected. Without deflation, it would be very

\footnotetext{
*nicolas.boulle@maths.ox.ac.uk

†echarala@calpoly.edu

patrick.farrell@maths.ox.ac.uk

§kevrekid@math.umass.edu
}

difficult to identify these complex (literally and figuratively) topological stationary points of the infinite-dimensional energy landscape. In fact, as we increase the atom number of the system, we observe this complexity to be substantially enhanced, leading to states which, while stationary, are not straightforwardly decomposable into simpler linear or nonlinear building blocks. To further investigate the nature of the identified solutions, we compute their spectral linearization (so-called Bogoliubov-de Gennes) modes, and conduct transient simulations of prototypical unstable states to explore the dynamical behavior of their instabilities. The present work showcases, in our view, the utility and potential impact of the deflation method to complex 3D physical problems well beyond atomic BECs.

The structure of this paper is as follows. In Sec. II, we present the model and computational techniques employed in this work. Our numerical results on the existence, stability, and selected transient simulations of nonlinear excitations are demonstrated in Sec. III. Section IV summarizes our findings and presents directions for future study.

\section{THEORETICAL AND NUMERICAL SETUP}

The 3D NLS/GP model of interest is of the form [4-6]:

$$
i \psi_{t}=-\frac{1}{2} \nabla^{2} \psi+|\psi|^{2} \psi+V(\mathbf{r}) \psi,
$$

subject to homogeneous Dirichlet conditions on the boundary of the domain $D=[-6,6]^{3}$. Here, $\psi=\psi(\mathbf{r}, t)$ plays the role of the suitably normalized (see Ref. [6] for details) wave function, while $V$ is the external confining potential of the form $V(\mathbf{r})=\frac{1}{2} \Omega^{2}|\mathbf{r}|^{2}$, a spherically symmetric trap of strength $\Omega$, which we fix to $\Omega=1$. The boundary conditions do not affect the solutions for this choice of the trap strength since the domain is chosen large enough so that the solutions vanish well before reaching the boundary. Using the standard standing wave decomposition $\psi(\mathbf{r}, t)=e^{-i \mu t} \phi(\mathbf{r})$ (where $\mu>0$ is the chemical potential), we obtain the stationary NLS/GP elliptic 
problem of the form:

$$
F(\phi) \doteq-\frac{1}{2} \nabla^{2} \phi+|\phi|^{2} \phi+V(\mathbf{r}) \phi-\mu \phi=0 .
$$

This equation is discretized using piecewise cubic Lagrange finite elements on a structured hexahedral grid using the Firedrake finite element library [22]. Multiple solutions to the discretized problem are sought using deflation, which we briefly describe here.

Suppose that Newton's method has discovered an isolated root $\phi_{1}$ of $F$. Deflation constructs a new problem $G$ via

$$
G(\phi) \doteq\left(\frac{1}{\left\|\phi-\phi_{1}\right\|^{2}}+1\right) F(\phi)
$$

where $\|\cdot\|$ is a suitable norm, in this case the $H^{1}$ norm. The essential idea is that $\left\|\phi-\phi_{1}\right\|^{2}$ approaches 0 faster than $F(\phi)$ does as $\phi \rightarrow \phi_{1}$, hence avoiding the convergence to $\phi_{1}$ of a Newton iteration applied to $G$. The addition of 1 ensures that $G(\phi) \approx F(\phi)$ far from $\phi_{1}$. By applying Newton's method to $G$, an additional root $\phi_{2} \neq \phi_{1}$ can be found, and the process repeated (by premultiplying with additional factors) until Newton's method fails to converge from the available initial guesses.

Previous applications of deflation to the study of BECs in $2 \mathrm{D}$ were combined with continuation in $\mu$, capturing solutions as they bifurcate from known ones [20,21]. This strategy is too expensive in 3D and so a different approach is taken here. We fix $\mu=6$ and exploit the linear (low-density, i.e. $|\phi|^{2} \rightarrow 0$ ) limit states to furnish a large number of initial guesses for Newton's method. The algorithm proceeds as follows. Given an initial guess, the inner loop applies Newton's method and deflation until no more solutions are found. The outer loop iterates over the available initial guesses, and terminates when no guess yields any solutions. We emphasize that at each application of Newton's method, all previously computed solutions are deflated, to avoid their rediscovery.

The initial guesses used were the eigenstates of the linear limit in Cartesian, cylindrical and spherical coordinates. The Cartesian eigenstates are given by

$$
|k, m, n\rangle \doteq H_{k}(\sqrt{\Omega} x) H_{m}(\sqrt{\Omega} y) H_{n}(\sqrt{\Omega} z) e^{-\Omega r^{2} / 2},
$$

with associated energy (i.e., chemical potential) $E_{k, m, n} \doteq(k+$ $m+n+3 / 2) \Omega$. The $H_{k, m, n}$ in Eq. (4) stand for the Hermite polynomials and $k, m$, and $n$ are non-negative integers. The cylindrical eigenstates are given by

$$
|K, l, n\rangle_{\mathrm{cyl}} \doteq q_{K, l}(R) e^{i l \theta} H_{n}(\sqrt{\Omega} z) e^{-\Omega\left(R^{2}+z^{2}\right) / 2},
$$

with $E_{K, l, n} \doteq(2 K+|l|+n+3 / 2) \Omega$ where $K, n$ are nonnegative integers, and $l=0, \pm 1, \pm 2, \ldots$ The radial profile $q_{K, l}$ in (5) is given by $q_{K, l} \sim r^{l} L_{K}^{l}\left(\Omega R^{2}\right) e^{-\Omega R^{2} / 2} \Omega$ where $L_{K}^{l}$ are the associated Laguerre polynomials in $R=\sqrt{x^{2}+y^{2}}$.

Finally, the spherical eigenstates are given by $|K, l, m\rangle_{\mathrm{sph}}$, where the radial part is similar but now in the spherical variable $r=\sqrt{x^{2}+y^{2}+z^{2}}$, and the angular part is described by the spherical harmonics $Y_{l m}(\theta, \phi)$ with $E_{K, l, m}=(2 K+l+$ $3 / 2) \Omega$. The quantum numbers $K$ and $l$ are nonnegative integers and $m=0, \pm 1, \ldots, \pm l$. All these states with $E \leqslant \mu=6$ were used in the process described above.

Once a solution has been discovered, the next step is the consideration of the spectral stability of the solutions via the well-established [4-6] Bogoliubov-de Gennes (BdG) analysis. More specifically, we assume the following perturbation ansatz around a stationary solution $\phi^{0}$ :

$$
\tilde{\psi}(\mathbf{r}, t)=e^{-i \mu t}\left\{\phi^{0}(\mathbf{r})+\epsilon\left[a(\mathbf{r}) e^{i \omega t}+b^{*}(\mathbf{r}) e^{-i \omega^{*} t}\right]\right\},
$$

where $\epsilon$ is a (formal) small perturbation parameter, $\omega$ is the eigenfrequency, and $(a, b)^{\top}$ the corresponding eigenvector. After substituting Eq. (6) into the time-dependent NLS equation [cf., Eq. (1)] we obtain the following complex eigenvalue problem:

$$
\left(\begin{array}{cc}
A_{11} & A_{12} \\
-A_{12}^{*} & -A_{11}
\end{array}\right)\left(\begin{array}{l}
a \\
b
\end{array}\right)=\rho\left(\begin{array}{l}
a \\
b
\end{array}\right)
$$

where $\rho=-\omega$ and the matrix elements are given by

$$
\begin{aligned}
& A_{11}=-\frac{1}{2} \nabla^{2}+2\left|\phi^{0}\right|^{2}+V(\mathbf{r})-\mu, \\
& A_{12}=\left(\phi^{0}\right)^{2} .
\end{aligned}
$$

We solve the above eigenvalue problem for the eigenfrequencies $\omega$ and eigenvectors $(a, b)^{\top}$ by using a Krylov-Schur algorithm with a shift-and-invert spectral transformation [23], implemented in the SLEPc library [24] [details about the decomposition of Eq. (7) into real and imaginary parts are presented in the Appendix]. Upon convergence of the eigenvalue solver, we draw conclusions on the stability characteristics of the stationary state $\phi^{0}$, i.e., real $\omega$ implies stability (vibrations), while complex $\omega$ is associated with instability.

Finally, we explore the dynamical evolution of unstable solutions via transient numerical simulations of Eq. (1). To that end, let $\phi^{0}$ be an unstable stationary solution discovered by deflation, and $(a, b)^{\top}$ be its most unstable eigendirection normalized according to

$$
\int_{D}\left(|a|^{2}+|b|^{2}\right) d x=1
$$

We integrate Eq. (1) forward in time until $t=50$ using the following perturbed solution as initial state

$$
\psi(x, y, z, t=0)=\phi^{0}+\epsilon\left[a+b^{*}\right],
$$

thus perturbing $\phi^{0}$ along its most unstable eigendirection with perturbation parameter $\epsilon$ chosen to be 0.1 . Next, let $\Delta t$ be the time step-size $\left(\Delta t=5 \times 10^{-2}\right.$ in this work) such that $t_{n}=$ $n \Delta t$ and $\psi^{(n)} \doteq \psi\left(\mathbf{r}, t_{n}\right)$ with $n \geqslant 0$. Then, for given $\psi^{(n)}$ at the $n$th time step, $\psi^{(n+1)}$ is obtained (implicitly) by a modified Crank-Nicolson method [25]:

$$
\begin{aligned}
i \frac{\psi^{(n+1)}-\psi^{(n)}}{\Delta t}= & \left(-\frac{1}{2} \nabla^{2}+V(\mathbf{r})+\frac{1}{2}\left(\left|\psi^{(n+1)}\right|^{2}+\left|\psi^{(n)}\right|^{2}\right)\right) \\
& \times \frac{\psi^{(n+1)}+\psi^{(n)}}{2},
\end{aligned}
$$

where cubic finite elements are employed for the spatial discretization as before. At each time step $n$, a nonlinear problem is solved by using Newton's method. It is pointed out in passing that the time-marching scheme employed in this work [cf., Eq. (11)] preserves both the squared $L^{2}$ norm (i.e., atom number)

$$
N(\psi) \doteq \int_{D}|\psi|^{2} d x
$$



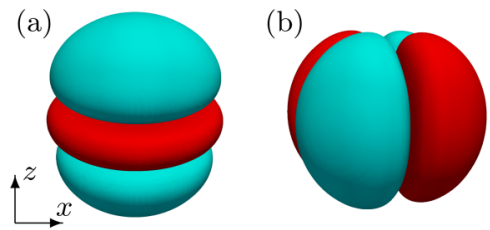

(c)
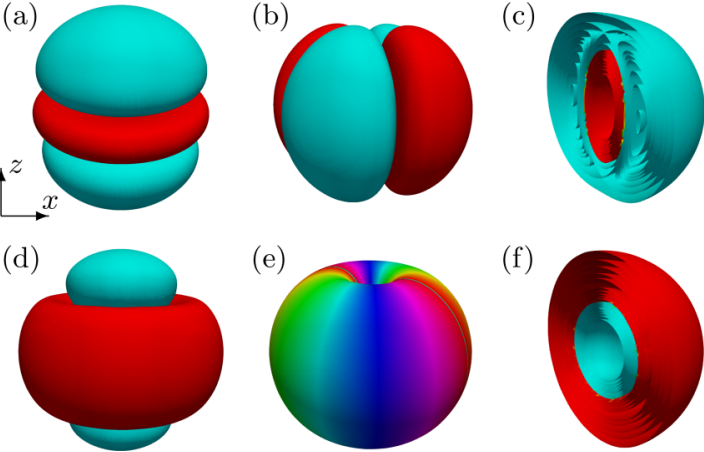

(f)

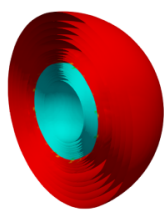

FIG. 1. Some solutions obtained by deflation that emanate from the second eigenvalue of the linear spectrum at $\mu=7 / 2$. The colors represent the argument of the solutions, ranging from $-\pi$ to $\pi$ (blue and red represent a phase of 0 and $\pm \pi$, respectively). The states in panels (a)-(d) and (f) are real, while (e) is complex.

and the energy of the solutions

$$
\mathcal{E}(\psi)=\int_{D}\left(\frac{1}{4}|\nabla \psi|^{2}+\frac{1}{2} V(\mathbf{r})|\psi|^{2}+\frac{1}{4}|\psi|^{4}\right) d x,
$$

to machine precision. We now turn to discussing the solutions obtained through the application of these numerical methods for the 3D NLS/GP problem.

\section{NUMERICAL RESULTS}

We briefly describe the physical meaning of the quantum numbers for the Cartesian, cylindrical, and spherical states, as they are useful in what follows. In the case of the Cartesian eigenfunctions, the quantum numbers $k, m$, and $n$ are simply the numbers of cuts along the $x, y$, and $z$ axes, respectively. For instance, in Fig. 1, panel (a) represents a $|0,0,2\rangle$ Cartesian state with 2 cuts along the $z$ axis (and $\pi$ phase differences across them), while panel (b) is $|1,1,0\rangle$, bearing one planar cut along the $x$ axis, and one along the $y$ axis. Combinations of states are also possible, such as the one in panel (c) of $|2,0,0\rangle+r|0,2,0\rangle+|0,0,2\rangle$ (in the particular example of this panel $r \approx 3.39$ ), which forms a $2 \mathrm{D}$ ring along the $y$ and $z$ axes embedded in 3D space.

Vortical structures and rings can be identified in the cylindrical system of coordinates. Here, $K$ denotes the number of cylindrical (nodal) surfaces, $l$ the topological charge of the configuration and $n$ the number of planar cuts along the $z$ axis. For example, panel (d) of Fig. (1) is a so-called ring dark soliton state (extended in 3D) $|1,0,0\rangle_{\text {cyl }}$ that has been recently considered in Ref. [26], and panel (e) is the $|0,2,0\rangle_{\text {cyl }}$ state, i.e., a vortex line, piercing through the BEC with topological charge $l=2$.

Finally, in the spherical representation, $K$ denotes the number of spherical (nodal) shells within the solution, $l-m$ denotes the number of planar cuts along the $z$ axis, and $m$ denotes the topological charge of vortical lines. Panel (f) is the $|1,0,0\rangle_{\text {sph }}$ state corresponding to a spherical shell dark solitary wave, which is also connected with recent work [27].

The ground state of the system (starting at $\mu=3 / 2$ ) is known to always be spectrally and nonlinearly stable [4,5]. The case of the first excited states (e.g., dipolar states and
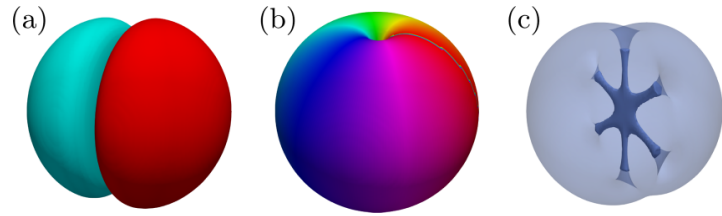

FIG. 2. Solutions emanating from the first excited state $\mu=5 / 2$ are shown in panels (a) and (b). In particular, panels (a) and (b) show a dipole and single vortex line solution. The colors represent the argument of the solutions, ranging from $-\pi$ to $\pi$ (blue and red represent a phase of 0 and $\pm \pi$, respectively). Panel (c) also emanates from the first excited state and corresponds to the density isosurfaces of the Chladni soliton at densities 0.30 and 0.35 .

single vortex lines) emanating from $\mu=5 / 2$ is interesting but reasonably well understood on the basis of corresponding 2D studies [6], since no fundamentally novel states appear to emerge in 3D. Indicatively, Fig. 2(a) shows a $|1,0,0\rangle$ Cartesian state with one cut along the $x$ axis whereas Fig. 2(b) presents the $|0,1,0\rangle_{\text {cyl }}$ state corresponding to a single vortex line with topological charge $l=1$. The rotations of these solutions along the $x, y$, and $z$ axes such as the $|0,1,0\rangle$ and $|0,0,1\rangle$ Cartesian states are also obtained by deflation but are not reported. A typical example of a relevant solution is shown in Fig. 2(c) and represents a so-called Chladni soliton, previously identified in cylindrical geometry in [15,28]. However, the states that follow next are sufficiently complex to feature the emergence of unexpected patterns, yet it will still be possible to connect them to fundamental building blocks of topological patterns such as vortex lines and rings [14].

We thus focus our discussion on states emanating from the second excited state of the linear problem at $\mu=7 / 2$. To that end, steady-state solutions to the NLS equation are identified by the deflation method at $\mu=6$ (all the solutions presented in this paper are displayed at $\mu=6$ ). The branches are then continued backward in $\mu$ down to the linear limit by a standard zero-order continuation method [[29], Sec. 4.4.2].

Examples of these solutions are shown in Fig. 3. In this figure, we observe that deflation enables us to converge to states with multiple coherent structures such as the one of panel (a) consisting of a vortex line and a planar dark soliton. The linear state corresponding to such a nonlinear wave form is $|0,1,1\rangle_{\mathrm{cyl}}$. This nonlinear state undertakes a symmetry-
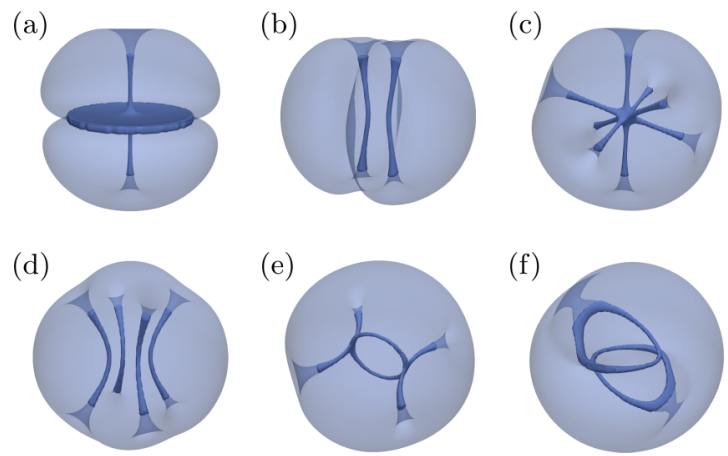

FIG. 3. More exotic solutions discovered by deflation that emanate from the second excited state. The panels show density isosurfaces of the states at densities 0.30 and 0.35 . 

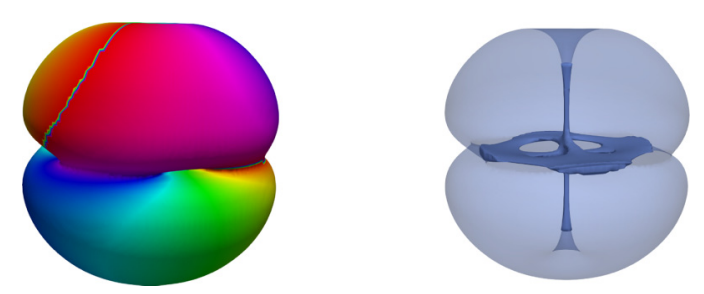

FIG. 4. Left: argument of the solution at $\mu=6$. This branch bifurcates from the state of Fig. 3(a) at $\mu \approx 5.84$ (see Fig. 5). Right: density isosurfaces of the state at densities 0.30 and 0.35 .

breaking bifurcation at $\mu \approx 5.84$ and gives birth to the wave form of Fig. 4. However, more complex multivortex topological states can progressively be identified as well. Panel (b) of Fig. 3 represents a pair of vortex lines: at the linear limit such a state can be formulated as the linear combination $|1,1,0\rangle+i|0,2,0\rangle$, in line with what is known about vortex dipole bifurcations [6]. Panel (c) represents what was termed a vortex star in [30], arising at the linear limit via the linear combination $|2,0,0\rangle-|0,2,0\rangle+i[|2,0,0\rangle-|0,0,2\rangle]$. Panel (d) shows a generalization of the well-known 2D vortex quadrupole [31] consisting of four bent alternating charge vortex lines.

The solutions in Figs. 3(a)-3(d) either allow for a direct tracing of their linear limit or have been previously identi- fied. However, deflation allows us to go well beyond these. Important examples of this arise in panels (e) and (f) of Fig. 3. Panel (e) consists of a vortex ring combined with two (oppositely charged) vortex line "handles". This state, too, can be identified at the linear limit through a more complex topologically charged combination, as $|2,0,0\rangle+|0,2,0\rangle+i|1,0,1\rangle$. Even more complex is the state in panel (f), which does not bear a linear analog. This state involves two vortex rings, both of which are bent; i.e., instead of having two "perpendicular" vortex rings (e.g., in the $x y$ and $y z$ planes), it is as if the top half of the one has connected itself with the right half of the other, and the bottom half of one with the left half of the other. This configuration was discovered by deflation at $\mu=6$ but the branch terminates by $\mu=5.9$ without ever reaching the linear limit of $\mu=7 / 2$. In other words, this appears to be a purely nonlinear state not derivable by some suitable combination of linear eigenstates. We conclude the presentation of our numerical results for the second excited states by presenting the associated bifurcation diagram in Fig. 5 where the total number of atoms [cf., Eq. (12)] is used as the diagnostic functional. The inset panel in the top-left corner therein uses the atom-number-difference $\Delta N$ between the branches Figs. 3(a) and 4 to illustrate a bifurcation in the diagram.

We now explore the BdG spectral stability of selected solutions (the spectra of all states shown in Figs. 1 and 3 are

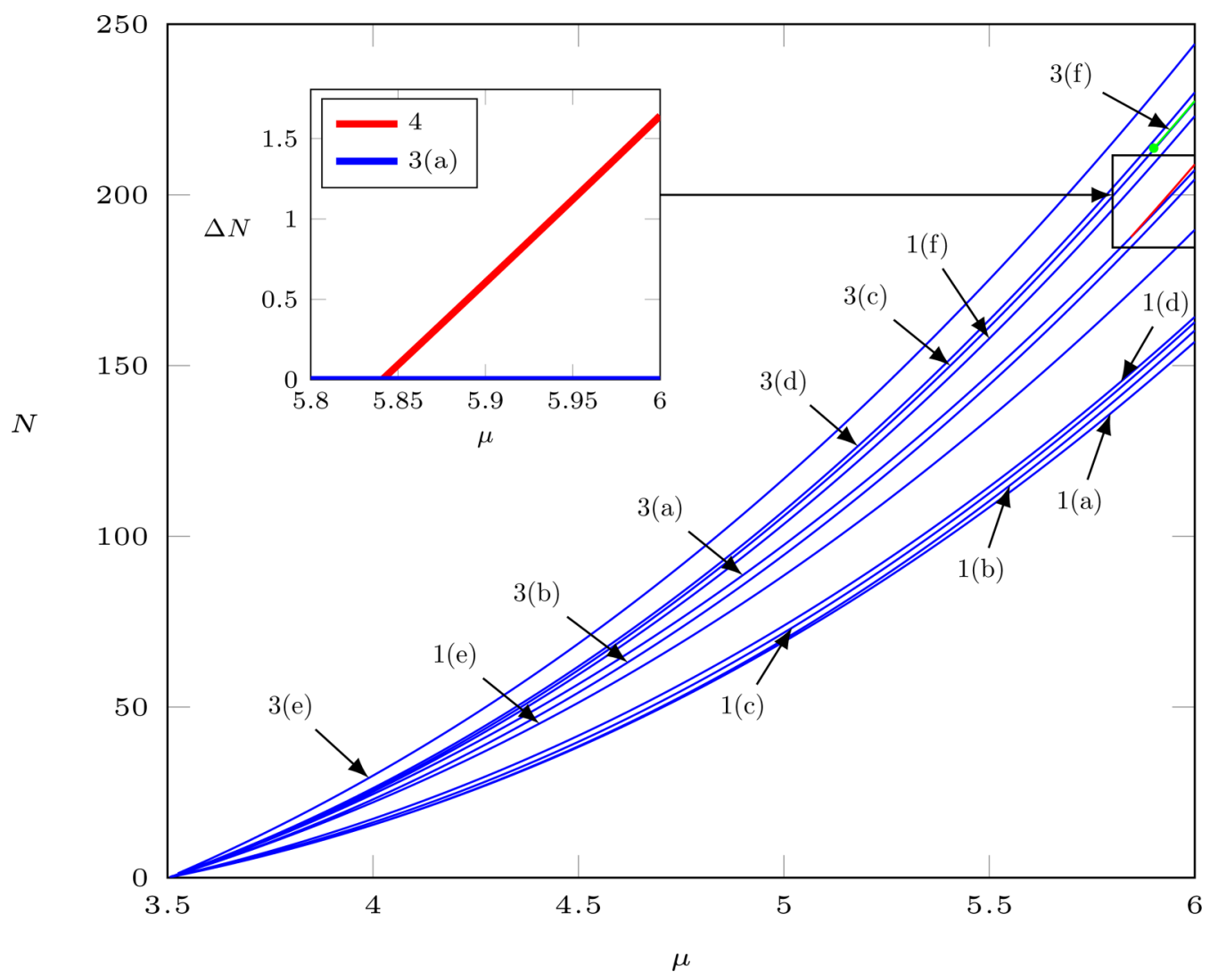

FIG. 5. Bifurcation diagram of the solutions emanating from the second excited state at $\mu=7 / 2$. The labels indicate the solutions represented in the different panels of Figs. 1 and 3. The main panel corresponds to the total number of atoms $N$ as a function of $\mu$, while the top-left inset shows the atom number difference $\Delta N$ between the branch Figs. 3(a) and 4, colored in red. The green branch illustrates the solution displayed in Fig. 3(f) and its terminal point at $\mu=5.9$. 

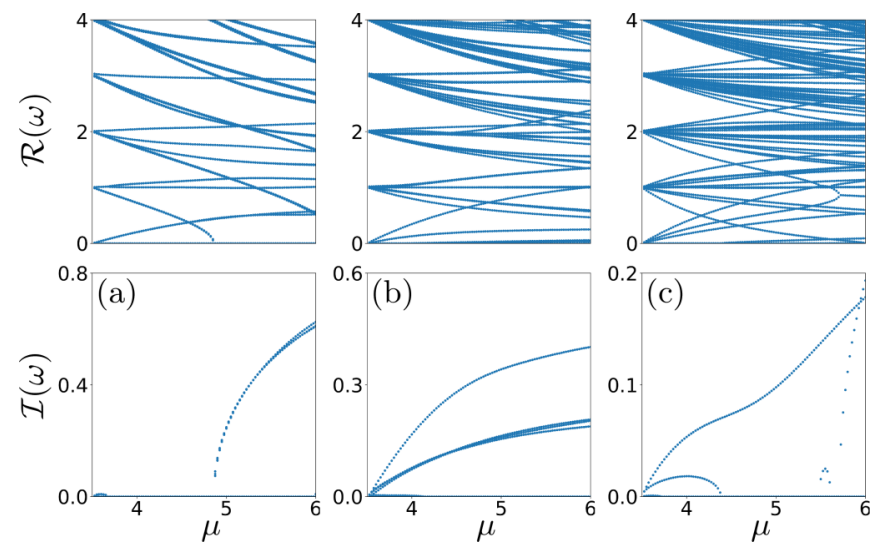

FIG. 6. Spectra of (a) the spherical shell dark solitary wave presented in Fig. 1(f); (b) the vortex star presented in Fig. 3(c); (c) the vortex ring with two vortex lines presented in Fig. 3(e). The real and imaginary parts of the corresponding eigenfrequencies $\omega$ are depicted in the top and bottom panels respectively.

presented in Figs. 10 and 11 in the Appendix, respectively). In fact, some of the identified wave forms are dynamically robust for an interval within their existence range. An example of this form is the spherical shell dark soliton of Fig. 1(f) with its spectrum presented in Fig. 6(a). However, most are indeed dynamically unstable, as expected; see, e.g., the case of the vortex star in Fig. 6(b). Interestingly, our BdG computations reveal that it is not the case that the most complex states are also the most unstable ones (see Fig. 6). An example of this type can be found in the vortex ring-double vortex line state of Fig. 3(e) with spectrum presented in Fig. 6(c). While the solution is highly complex, it only bears a single unstable mode for a wide parametric interval, and at most bears two over the interval studied. Even more importantly, in our dimensionless units (scaled by the harmonic trapping frequency), the relevant growth rate is typically of the order of $0.1-0.2$. This means that the characteristic dimensionless growth time is about 5-10 oscillation times, and if the perturbation is sufficiently small the instability manifestation time can be expected to be 2-3 times larger, in line with the dynamical observations to which we now turn.

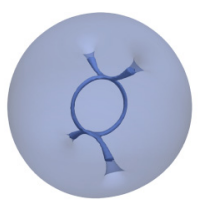

$t=15$

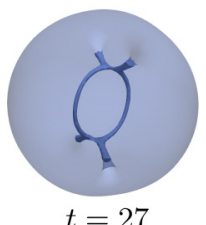

$t=27$

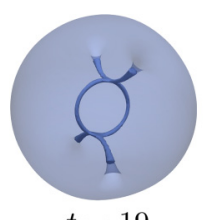

$t=19$

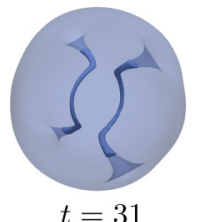

$t=31$

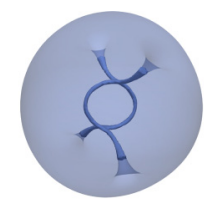

$t=23$

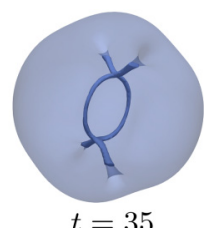

$t=35$
FIG. 7. Snapshots of the vortex ring-double vortex line state obtained by solving the time-dependent NLS equation. The steady-state solution of panel (e) in Fig. 3 is initially perturbed along its dominant unstable eigenmode.
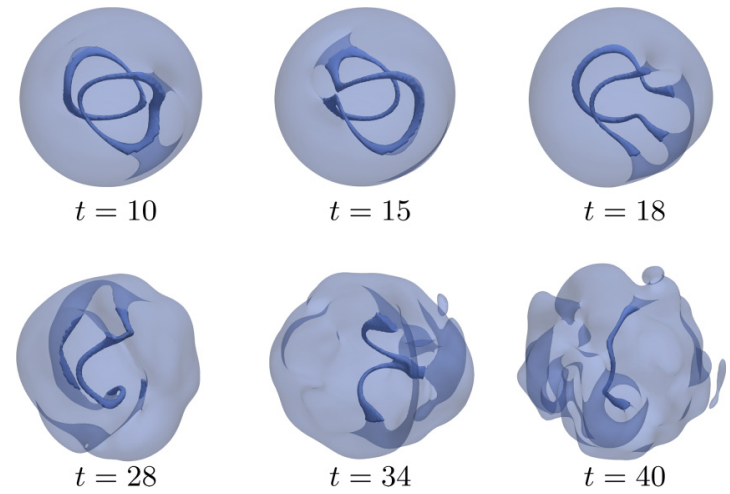

FIG. 8. Snapshots of the two vortex rings state of Fig. 3(f), obtained by solving the time-dependent NLS equation with the modified Crank-Nicolson time-stepping scheme.

We employ the Crank-Nicolson method to explore the dynamical implications of these instabilities. The initial states are prepared according to Eq. (10). In the case of Fig. 3(e), we observe in the snapshots of the evolution of Fig. 7 that the vortex ring and two vortex lines break up into two vortex lines that are strongly bent (in fact, they are somewhat reminiscent of the U-shaped vortex lines of [32]). After about 20-30 dimensionless time units-in line with our eigenvalue predictions - the configuration is characterized by splittings and reconnections (including ones reformulating the original configuration). We have performed similar computations for other complex, topological states such as that shown in
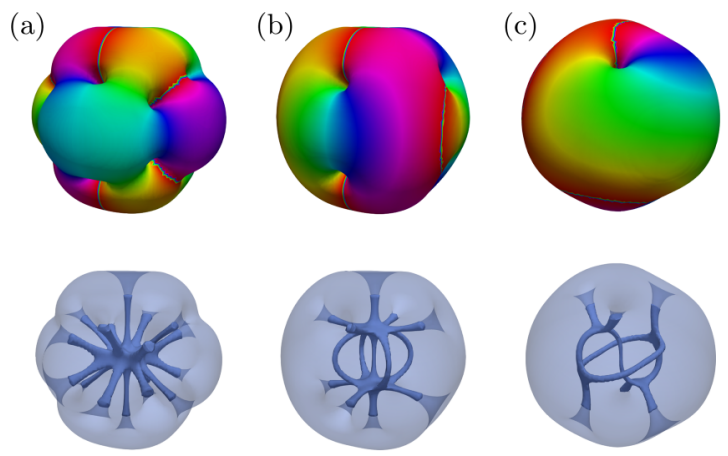

(d)
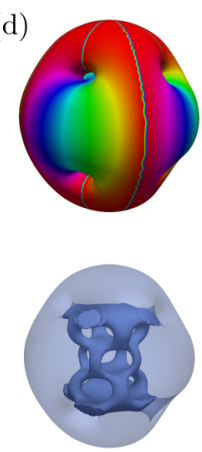

(e)
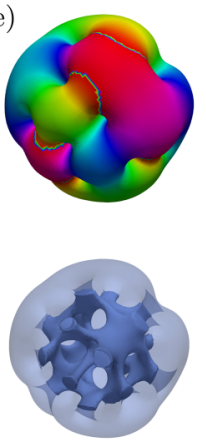

(f)
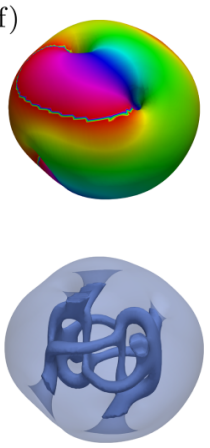

FIG. 9. Some of the solutions discovered by deflation that emanate from the third [panels (a)-(c)] and fourth [panels (d)-(f)] excited states at $\mu=9 / 2$ and $\mu=11 / 2$. The first and third rows show the phase of the solutions while the second and fourth rows represent the density isosurfaces at densities 0.30 and 0.35 . 

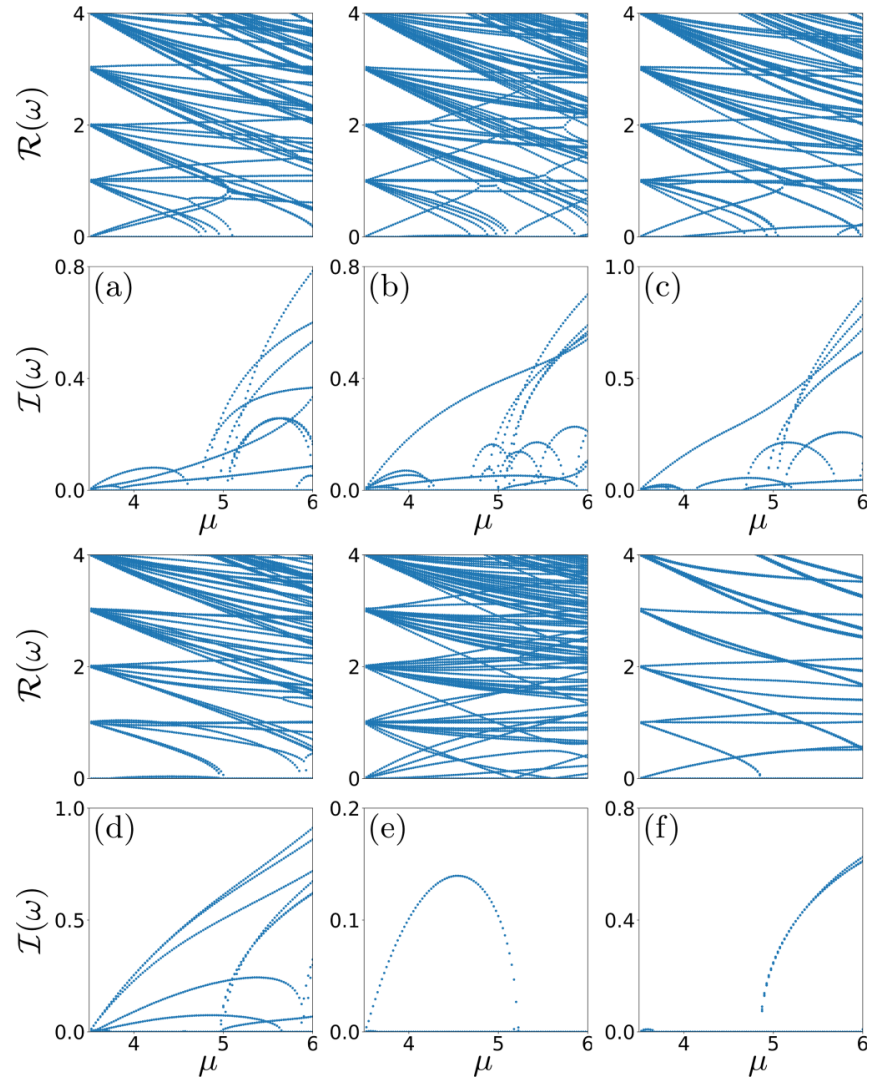

FIG. 10. Spectra of the solutions presented in Fig. 1 [e.g., panel (a) corresponds to the branch 1(a), illustrated in panel (a) of Fig. 1] . The real and imaginary parts of the corresponding eigenfrequencies $\omega$ are, respectively, depicted in the top and bottom panels.

Fig. 3(f), showcasing in some such cases more radical dynamical breakups, i.e., the eventual persistence of a single, strongly excited vortex line; see relevant snapshots in Fig. 8 and the movies in [33]. Importantly, we note that the relevant time scales for standard choices of the trap strength are on the order of hundreds of milliseconds, and hence the configurations are expected to be well within windows of experimental accessibility. Furthermore, even when the configurations become unstable, as in the dynamics of Fig. 7, they appear to result in oscillatory dynamics reconstructing the relevant states in a nearly periodic fashion for far longer times (rather than dispersing or yielding chaotic dynamics). This further enhances the potential observability window of the states of interest.

We close our presentation of the numerical results of deflation by offering a glimpse into the capabilities of the method for discovering higher excited states, i.e., ones that are initiated not from the second (as up to now), but rather from the third and fourth excited states at $\mu=9 / 2$ and $\mu=11 / 2$, respectively. Some of the relevant nonlinear states discovered with deflation can be found in Fig. 9. The first examples, such as those of panels (a) and (b) can be identified straightforwardly: panel (a) represents a nonlinear configuration bearing nine vortex lines in a generalization of the star-shaped configuration of Fig. 3(c) and [30]. The state of Fig. 9(b) appears to be a configuration bearing two perpendicular vortex rings
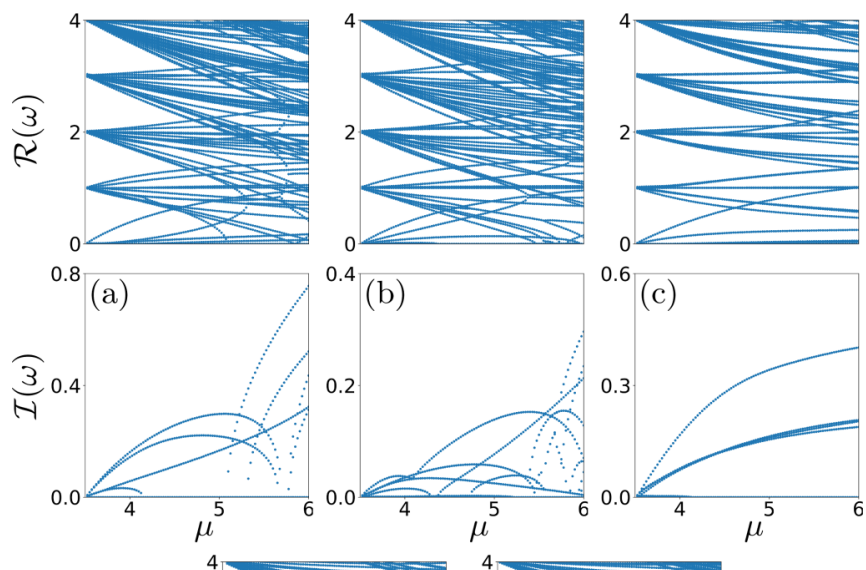

0.4 (b)

(b)

$0.6(\mathrm{c})$
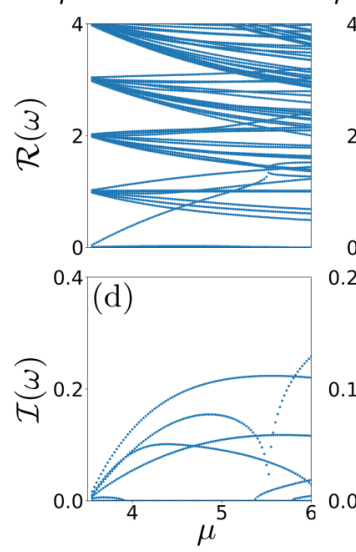

$0.2(\mathrm{e})$

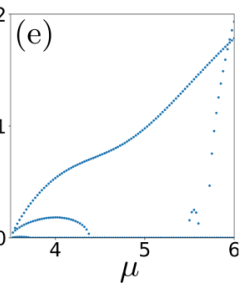

FIG. 11. Spectra of the solutions presented in Figs. 3(a)-(e).

[now joined - cf., panel Fig. 3(f)], along with five vortex lines: four of these are tangent, similar to the ones of Fig. 3(e), while one is piercing through the planes of the two rings. Going beyond these, however, the states become highly complex. Fig. 9(c) shows what appears to be a combination of an Sshaped and two U-shaped vortex lines (in the terminology of Ref. [32]) along with a clearly discernible vortex ring. Labyrinthine patterns of conjoined vortex rings and vortex lines appear; at the moment we do not have an immediate classification. Figure 9(d) displays an apparent lattice of vortex rings, while panel Fig. 9(e) is reminiscent of the vortex ring cages that appear in the dynamical instabilities of other states such as the spherical dark shell solitary wave of Fig. 1(f) [27]. Figure 9(f) displays a conglomeration of bent vortex lines.

\section{CONCLUSIONS AND FUTURE CHALLENGES}

Deflation reveals unknown and intriguing dynamical states of a fundamental model for 3D Bose-Einstein condensates. By building a priori knowledge of the linear eigenstates into the deflation procedure, we are able to identify a wide range of solutions. Many of the solutions found can be characterized using these underlying linear limits. However, deflation can also discover numerous unexpected topological nonlinear states such as the vortex ring with two vortex lines, or the coupled bent vortex rings of Fig. 3. Despite their complexity, such states may only be weakly unstable (thus potentially tractable experimentally) and feature long-time dynamics consisting of splittings and recombinations towards the original state. Recent experimental advances have enabled the formulation 
(painting) of arbitrary potentials [34], the establishment of arbitrary density [35] or imposition of controlled phase [36-38] patterns, and even the realization of unstable (but sufficiently long-lived) complex topological states such as vortex knots [39]. In light of all these developments and their impact on vortex ring and line dynamics [40-42], we expect that the states identified in this work to be within reach of current state-of-the-art experimental efforts.

As the nonlinearity of the model is increased, so is the complexity of the available topological states; yet the numerical methods discussed here appear to remain efficient in this regime. They reveal not only solutions that are generalizations of previous ones, but also vortex ring lattices, cages, bent-connected-multivortex rings, and line patterns, and more. These warrant further study, topological classification and deeper physical understanding. We believe that this technique paves the way for a wide range of future exciting explorations in this and related fields.

\section{ACKNOWLEDGMENTS}

This work is supported by the EPSRC Centre For Doctoral Training in Industrially Focused Mathematical Modelling (EP/L015803/1) in collaboration with Simula Research Laboratory (NB), EPSRC Grants No. EP/R029423/1 and No. EP/V001493/1 (PEF), and by the U.S. National Science Foundation under Grant No. PHY-1602994 (PGK).

\section{APPENDIX: BdG SPECTRAL DECOMPOSITION}

We decompose the steady-state solution $\phi^{0}$, eigenvector $(a, b)^{\top}$, and eigenvalue $\rho$ into real and imaginary components as $\phi^{0}=\phi_{r}^{0}+i \phi_{c}^{0}, a=a_{r}+i a_{c}, b=b_{r}+i b_{c}$, and $\rho=\rho_{r}+$ $i \rho_{c}$, respectively, and rewrite Eq. (7) as

$$
\begin{array}{r}
\left(\begin{array}{cccc}
A_{11} & 0 & B_{1} & -B_{2} \\
0 & A_{11} & B_{2} & B_{1} \\
-B_{1} & -B_{2} & -A_{11} & 0 \\
B_{2} & -B_{1} & 0 & -A_{11}
\end{array}\right)\left(\begin{array}{l}
a_{r} \\
a_{c} \\
b_{r} \\
b_{c}
\end{array}\right) \\
=\left(\begin{array}{cccc}
\rho_{r} & -\rho_{c} & 0 & 0 \\
\rho_{c} & \rho_{r} & 0 & 0 \\
0 & 0 & \rho_{r} & -\rho_{c} \\
0 & 0 & \rho_{c} & \rho_{r}
\end{array}\right)\left(\begin{array}{l}
a_{r} \\
a_{c} \\
b_{r} \\
b_{c}
\end{array}\right),
\end{array}
$$

where $B_{1}=\left(\phi_{r}^{0}\right)^{2}-\left(\phi_{c}^{0}\right)^{2}$ and $B_{2}=2 \phi_{r}^{0} \phi_{c}^{0}$. The eigenvalues of the matrix on the right-hand side of Eq. (A1) are $\rho_{r} \pm i \rho_{c}$ (with multiplicity two). Therefore, solving a real eigenvalue problem with the left-hand matrix of Eq. (A1) yields the same eigenvalues and eigenvectors as the complex eigenvalue problem (7). We use a Krylov-Schur algorithm with a shiftand-invert spectral transformation [23], implemented in the SLEPc library [24], to solve the following eigenvalue problem:

$$
\left(\begin{array}{cccc}
A_{11} & 0 & B_{1} & -B_{2} \\
0 & A_{11} & B_{2} & B_{1} \\
-B_{1} & -B_{2} & -A_{11} & 0 \\
B_{2} & -B_{1} & 0 & -A_{11}
\end{array}\right)\left(\begin{array}{l}
a_{r} \\
a_{c} \\
b_{r} \\
b_{c}
\end{array}\right)=\rho\left(\begin{array}{l}
a_{r} \\
a_{c} \\
b_{r} \\
b_{c}
\end{array}\right),
$$

where the matrices are real and $\rho=\rho_{r} \pm i \rho_{c}$ is complex. This problem is discretized with the same piecewise cubic finite element method used to find multiple solutions with deflation. The spectra of the states of Figs. 1 and 3 are shown in Figs. 10 and 11 , respectively.
[1] M. Ablowitz, B. Prinari, and A. Trubatch, Discrete and Continuous Nonlinear Schrödinger Systems (Cambridge University Press, Cambridge, 2004).

[2] M. Ablowitz, Nonlinear Dispersive Waves (Cambridge University Press, Cambridge, 2011).

[3] C. Sulem and P. Sulem, The Nonlinear Schrödinger Equation: Self-focusing and Wave Collapse (Springer, New York, 1999).

[4] C. J. Pethick and H. Smith, Bose-Einstein Condensation in Dilute Gases (Cambridge University Press, Cambridge, United Kingdom, 2002).

[5] S. Stringari and L. Pitaevskii, Bose-Einstein Condensation (Oxford University Press, Oxford, United Kingdom, 2003).

[6] P. Kevrekidis, D. Frantzeskakis, and R. Carretero-González, The Defocusing Nonlinear Schrodinger Equation (Society for Industrial and Applied Mathematics, Philadelphia, PA, 2015).

[7] A. Hasegawa and Y. Kodama, Solitons in Optical Communications (Clarendon Press, Oxford, 1995).

[8] M. Kono and M. Škorić, Nonlinear Physics of Plasmas (Springer-Verlag, Heidelberg, 2010).

[9] C. Kharif, E. Pelinovsky, and A. Slunyaev, Rogue Waves in the Ocean (Springer-Verlag, Berlin, 2009).

[10] D. J. Frantzeskakis, J. Phys. A 43, 213001 (2010).

[11] F. Abdullaev, A. Gammal, A. Kamchatnov, and L. Tomio, Int. J. Mod. Phys. B 19, 3415 (2005).
[12] A. L. Fetter and A. A. Svidzinsky, J. Phys.: Condens. Mater. 13, R135 (2001).

[13] A. L. Fetter, Rev. Mod. Phys. 81, 647 (2009).

[14] S. Komineas, Eur. Phys. J. Spec. Top. 147, 133 (2007).

[15] A. Muñoz Mateo and J. Brand, Phys. Rev. Lett. 113, 255302 (2014).

[16] J. Ruostekoski and J. R. Anglin, Phys. Rev. Lett. 86, 3934 (2001).

[17] J. Ruostekoski and J. R. Anglin, Phys. Rev. Lett. 91, 190402 (2003).

[18] D. Kleckner and W. Irvine, Nat. Phys. 9, 253 (2013).

[19] P. E. Farrell, A. Birkisson, and S. W. Funke, SIAM J. Sci. Comput. 37, A2026 (2015).

[20] E. G. Charalampidis, P. G. Kevrekidis, and P. E. Farrell, Commun. Nonlinear Sci. Numer. Simulat. 54, 482 (2018).

[21] E. Charalampidis, N. Boullé, P. Farrell, and P. Kevrekidis, Commun. Nonlinear Sci. Numer. Simulat. 87, 105255 (2020).

[22] F. Rathgeber, D. A. Ham, L. Mitchell, M. Lange, F. Luporini, A. T. T. Mcrae, G.-T. Bercea, G. R. Markall, and P. H. J. Kelly, ACM Trans. Math. Softw. 43, 1 (2016).

[23] G. W. Stewart, SIAM J. Matrix Anal. A. 23, 601 (2002).

[24] V. Hernandez, J. E. Roman, and V. Vidal, ACM Trans. Math. Softw. 31, 351 (2005). 
[25] M. Delfour, M. Fortin, and G. Payre, J. Comput. Phys. 44, 277 (1981).

[26] W. Wang, P. G. Kevrekidis, and E. Babaev, Phys. Rev. A 100, 053621 (2019).

[27] W. Wang, P. G. Kevrekidis, R. Carretero-González, and D. J. Frantzeskakis, Phys. Rev. A 93, 023630 (2016).

[28] A. M. Mateo and J. Brand, New J. Phys. 17, 125013 (2015).

[29] R. Seydel, Practical Bifurcation and Stability Analysis, Vol. 5 of Interdisciplinary Applied Mathematics (Springer, New York, 2010), 3rd ed.

[30] L.-C. Crasovan, V. M. Pérez-García, I. Danaila, D. Mihalache, and L. Torner, Phys. Rev. A 70, 033605 (2004).

[31] M. Möttönen, S. M. M. Virtanen, T. Isoshima, and M. M. Salomaa, Phys. Rev. A 71, 033626 (2005).

[32] A. Aftalion and I. Danaila, Phys. Rev. A 68, 023603 (2003).

[33] Please see the Supplemental Material at http://link.aps.org/ supplemental/10.1103/PhysRevA.102.053307 for movies of full time evolution corresponding to Figs. 7 and 8.

[34] K. Henderson, C. Ryu, C. MacCormick, and M. G. Boshier, New J. Phys. 11, 043030 (2009).
[35] G. Gauthier, I. Lenton, N. M. Parry, M. Baker, M. J. Davis, H. Rubinsztein-Dunlop, and T. W. Neely, Optica 3, 1136 (2016).

[36] M. J. H. Ku, W. Ji, B. Mukherjee, E. Guardado-Sanchez, L. W. Cheuk, T. Yefsah, and M. W. Zwierlein, Phys. Rev. Lett. 113, 065301 (2014).

[37] A. R. Fritsch, M. Lu, G. H. Reid, A. M. Piñeiro, and I. B. Spielman, Phys. Rev. A 101, 053629 (2020).

[38] S. Lannig, C.-M. Schmied, M. Prüfer, P. Kunkel, R. Strohmaier, H. Strobel, T. Gasenzer, P. G. Kevrekidis, and M. K. Oberthaler, Phys. Rev. Lett. 125, 170401 (2020).

[39] D. S. Hall, M. W. Ray, K. Tiurev, E. Ruokokoski, A. H. Gheorghe, and M. Möttönen, Nat. Phys. 12, 478 (2016).

[40] M. J. H. Ku, B. Mukherjee, T. Yefsah, and M. W. Zwierlein, Phys. Rev. Lett. 116, 045304 (2016).

[41] S. Serafini, M. Barbiero, M. Debortoli, S. Donadello, F. Larcher, F. Dalfovo, G. Lamporesi, and G. Ferrari, Phys. Rev. Lett. 115, 170402 (2015).

[42] S. Serafini, L. Galantucci, E. Iseni, T. Bienaimé, R. N. Bisset, C. F. Barenghi, F. Dalfovo, G. Lamporesi, and G. Ferrari, Phys. Rev. X 7, 021031 (2017). 